\title{
Study of Local and Distortional Stability of Thin-Walled Structures
}

\author{
Mahi Imene ${ }^{1}$, Djafour Naoual ${ }^{1}$ Djafour Mustapha $^{1}$ \\ ${ }^{1}$ Abou Bekr Belkaid, Civil Engineering, Tlemcen, Algeria
}

\begin{abstract}
Thin-walled structures have an increasingly large and growing field of application in the engineering sector, the goal behind using this type of structure is efficiency in terms of resistance and cost, however the stability of its components (the thin walls) remains the first aspect of the behavior, and a primordial factor in the design process. The hot rolled sections are known by a consequent post-buckling reserve, cold-formed steel sections which are thin-walled elements also benefit, in this case, it seems essential to take into account the favorable effects of this reserve in to the verification procedure of the resistance with respect to the three modes of failures of this type of structure. The design method that takes into account this reserve of resistance is inevitably the effective width method. The direct strength method has been developed to improve the speed and efficiency of the design of thin-walled profiles. The latter mainly uses the buckling loads (for Local, Distortional and Global mode) obtained from a numerical analysis and the resistance curves calibrated experimentally to predict the ultimate load of the profile. Among those, the behavior of a set of Cshaped profiles (highly industrialized) is studied, this type of section is assumed to be very prone to modes of local and distortional instability. The outcome of this investigation revealed very relevant conclusions both scientifically and practically.
\end{abstract}

\section{Introduction}

L'utilisation croissante des profilés à parois minces formées à froid réside dans la disponibilité et la facilité des procédés de fabrication qui ont donné la possibilité d'avoir des configurations diverses et plus optimisées afin de répondre aux objectifs structuraux et économiques. L'idée principale derrière l'utilisation de ce type de profilés est l'utilisation de la forme de la section au lieu de l'épaisseur pour résister aux efforts appliqués (1).

L'utilisation d'un matériau mince issu d'un processus de formage à froid induit à des considérations particulières dans la démarche de dimensionnement contrairement à une conception avec les éléments en acier laminé à chaud classique. Parmi les considérations majeures à entreprendre : les modes d'instabilité, l'effet du formage à froid et la résistance post critique (2).

\section{2 l'instabilité locale et distorsionnelle}

Étant donné que les composantes individuelles en acier formé à froid sont généralement assez minces par rapport à leur largeur, ces dernières si, elles sont soumises à la compression, ou à la flexion ou au cisaillement, elles peuvent être affectées par les modes d'instabilité à un niveau de contrainte inférieur à la limite d'élasticité, ces modes d'instabilités sont d'ordre local, global et distorsionnel comme le montre la figure1. En l'occurrence le mode local est l'un des principaux facteurs à considérer dans le dimensionnement. Il est bien évident que ce type d'éléments a un comportement et une réserve post critique assez appréciable ; et leur ruine ne se produit pas dès que la charge critique d'instabilité est atteinte, un chargement supplémentaire peut être encore supporté après l'apparition du mode local et avant que la charge ultime de l'élément soit atteinte (3).

$\mathrm{Au}$ cours de ces dernières années, le flambement distorsionnel a été considéré comme un état limite important pour la conception des poutres et des colonnes en acier formé à froid, ayant des semelles comprimées renforcées par des raidisseurs. Et par conséquent de nouvelles dispositions de conceptions ont été ajoutées dans les spécifications nord-Américaines actuelles. Le mode distortionnel peut aussi régir le comportement des éléments ayant un centre de gravité et un centre de cisaillement qui ne coïncident pas, ils sont toutefois enclins au flambement flexionnel-torsionnel qui peut être critique vu leur faible rigidité torsionnelle. Des études numériques et des investigations expérimentales ont montré l'existence d'une réserve poste critique pour le mode distorsionnel, mais elle est moins importante que celle du mode local (4). 


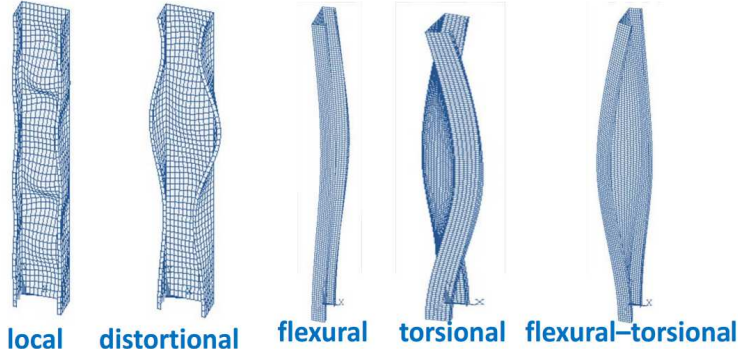

Fig. 1. les modes d'instabilité d'une colonne à parois minces de section en $\mathrm{C}$

\subsection{L'instabilité distorsionnelle}

C'est un mode qui implique le changement de la forme de la section transversale, cette déformation est manifestée par la rotation et la translation des coins arrondis et des lignes d'intersection entre les plaques formant la section du profilé, l'âme subit une flexion hors de son plan, alors que l'ensemble semelle-raidisseur se déplace en mode rigide. Ce mode se produit à une longueur d'onde intermédiaire entre celle du mode local et global. La figure2 présente la formé déformée du mode distorsionnel pour des sollicitations de compression et de flexion.

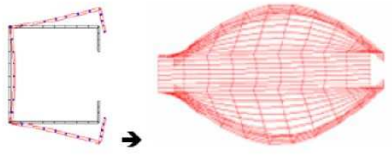

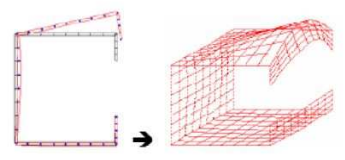

(b)
Fig. 2. La déformation d'une colonne en $\mathrm{C}$ en mode distorsionnel (a) sous la compression (b) sous la flexion

\subsubsection{Le calcul de la contrainte critique du mode distorsionnel}

Ce mode est le moins métrisé et le plus compliqué d'ailleurs c'est le dernier qui a été introduit dans les codes de calcul. La mise en évidence de la charge de ruine de ce mode de flambement se doit à Hancock et ses collaborateurs. En l'occurrence la charge critique de cette instabilité est exprimée dans les codes de calculs par les formules (1), (2) et (3) :

Pour le règlement Européen : la contrainte élastique du raidisseur vaut (5) :

$\sigma_{\mathrm{crs}}=2 .\left(\left(\mathrm{k} \cdot \mathrm{E} \cdot \mathrm{I}_{\mathrm{s}}\right)^{\wedge}(0.5)\right) / \mathrm{A}_{\mathrm{s}}$

$\mathrm{K}, \mathrm{A}_{\mathrm{s}}$, Is sont respectivement la rigidité du support, l'aire efficace, et le moment d'inertie de l'aire efficace.

Quant au règlement Américain et australien la contrainte critique est respectivement (6) (7) :

$\mathrm{F}_{\mathrm{cr}}=\left(\mathrm{k}_{\phi \mathrm{\phi f}}+\mathrm{k}_{\phi \mathrm{ew}}\right) /\left(\mathrm{k}_{\phi \mathrm{gf}}+\mathrm{k}_{\phi \mathrm{gw}}\right)$

$\mathrm{F}_{\mathrm{cr}}=\mathrm{E} / 2 \mathrm{~A}\left[\left(\alpha_{1}+\alpha_{2}\right)-\left(\left(\alpha_{1}+\alpha_{2}\right)^{2}+4 \alpha_{3}\right)^{(1 / 2)}\right.$

Ou $\mathrm{k}_{\phi e f}, \mathrm{k}_{\phi \mathrm{ew}}, \mathrm{k}_{\phi \mathrm{gf},} \mathrm{k}_{\phi \mathrm{gw}}$ sont les rigidités à la rotation élastiques et géométriques de la semelle et l'âme respectivement.
A est l'aire de la section du profilé, E le module d'Young, et $\alpha_{1}, \alpha_{2}, \alpha_{3}$ sont des paramètres qui tiennent compte des caractéristiques géométriques de la section.

\subsection{L'instabilité locale}

C'est le mode de flambement le plus fréquent des sections à parois minces, qui se produit en engendrant la déformation des parois planes de la section, sans aucune rotation ou translation des lignes d'intersection, cette déformation se manifeste par la flexion cylindrique de celles-ci (figure3), la longueur de demi-onde de ce mode est le premier minimum sur la courbe signature, définie comme étant plus petite, que la plus grande dimension de la section.

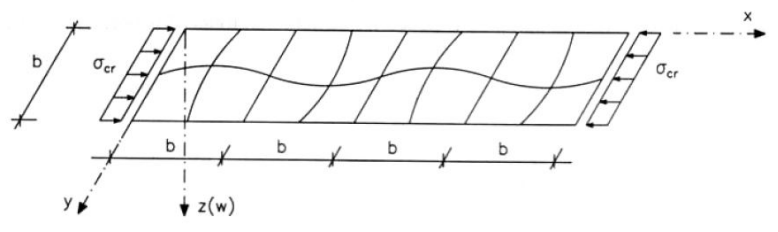

Fig. 3. Le voilement d'une plaque rectangulaire soumise à des contraintes longitudinales de compression

\subsubsection{Le calcul de la contrainte critique du mode local}

La contrainte critique de ce mode a été investiguée par Timoshenko et Gere 1961, et sa formulation dépend de l'élancement, du coefficient de voilement de la plaque, et de la longueur de demi-onde de ce mode (2).

En adoptant le concept de la méthode énergétique et la mise en œuvre de différentes substituions nécessaires, la contrainte critique du mode local est matérialisée par la formule (4) :

$\mathrm{F}_{\mathrm{crv}}=\mathrm{k}_{\mathrm{V}} \pi^{2} \mathrm{E} / 12 *\left(1-v^{2}\right)(\mathrm{t} / \mathrm{b})^{2}$

Où $\mathrm{Kv}$ est le coefficient de voilement, il dépend des conditions aux limites, du coefficient d'aspect et de la répartition de la contrainte. Plus la plaque est rigidifiée mieux est la valeur du coefficient du voilement et la contrainte du mode local, et par conséquent la performance de l'élément au mode local accroît. A cet effet vient l'intérêt de doter la paroi de raidisseur de bord, qui contribue à sa stabilité tout en empêchant son déplacement.

Du point de vue théorique l'élément se voile dès l'atteinte de sa charge critique, pour un chargement supplémentaire, la ruine ne se produit pas, et cela suite à une redistribution de contrainte, alors une charge additionnelle sera supportée, cette redistribution de contrainte a été idéalisée sur une partie de la parois appelée largeur efficace. C'est le comportement post critique par excellence car le reste de la paroi : les parties inefficaces sont présentes et collaborent également à la résistance. La méthode réglementaire qui prend en considération ce concept dans la procédure de design est la méthode de la largeur efficace (MLE) formulée par les équations (5), (6), (7) et (8). Introduite par Von Karman 
1932, Winter (1968) a apporté des améliorations et des modifications qui mettent en évidence les imperfections géométriques et les contraintes résiduelles de telle manière que :

$\mathrm{b}_{\mathrm{e}}=\mathrm{b} \quad$ si $\lambda \leq 0.673$

be $=\rho b \quad$ si $\lambda>0.673$

Où $\rho$ est le facteur de réduction de la largeur

$\rho=(1-0.22 / \lambda) / \lambda$ et $\quad \rho \leq 1$

$\lambda=1.052 /\left(\mathrm{k}_{\mathrm{v}}\right)^{1 / 2}(\mathrm{~b} / \mathrm{t})(\mathrm{f} / \mathrm{E})^{1 / 2}$

$\mathrm{f}$ peut être prise égale à $\mathrm{f}_{\mathrm{y}}, \lambda$ est l'élancement réduit, $\rho$ le facteur de réduction $b_{e} b$ la largeur effective et la largeur de la paroi respectivement.

\section{La nouvelle méthode de dimensionnement-la méthode de résistance directe}

Quand les sections acquièrent des formes complexes et optimisées, avec des raidisseurs de bords et intermédiaires, le processus de la méthode de la largeur efficace devient extrêmement compliqué et fastidieux en termes de calcul et du temps. En outre, la MLE ne tient pas compte de l'interaction qui existe entre les parois (l'âme et la semelle, semelle raidisseur). Afin de pallier ces problèmes la DSM a été développée par Pekoz et Schafer (1998), son concept consiste à éviter la décomposition de la section par la mise en évidence des courbes de résistances appliquées pour la section complète. Cette procédure s'appuie sur la même hypothèse empirique de MLE : la résistance ultime est en fonction de la charge élastique de flambement et la limite d'élasticité.

Les équations (9), (10), (11), (12), servent à évaluer la capacité portance en compression pour le mode local et distorsionnel

Pour $\lambda \leq 0.776 \quad \mathrm{P}_{\mathrm{nl}}=\mathrm{P}_{\mathrm{ne}}$

Pour $\lambda>0.776 \mathrm{P}_{\mathrm{nl}}=\left[1-0.15\left(\mathrm{P}_{\mathrm{crl}} / \mathrm{P}_{\mathrm{ne}}\right)^{0.4}\right]\left(\mathrm{P}_{\mathrm{crl}} / \mathrm{P}_{\mathrm{ne}}\right)^{0.4} \mathrm{P}_{\mathrm{ne}}$

Si $\lambda \leq 0.561 \quad \mathrm{P}_{\mathrm{nd}}=\mathrm{P}_{\mathrm{y}}$

Si $\lambda>0.561 \mathrm{P}_{\text {nd }}=\left[1-0.25\left(\mathrm{P}_{\text {crd }} / \mathrm{P}_{\mathrm{y}}\right)^{0.6}\right]\left(\mathrm{P}_{\text {crd }} / \mathrm{P}_{\mathrm{y}}\right)^{0.6} \mathrm{P}_{\mathrm{y}}$

\section{Les méthodes numériques d'analyse du comportement des profils à parois minces}

Les méthodes analytiques énoncées dans les standards de calculs sont calibrées par rapport aux résultats numériques et expérimentaux, cependant les ingénieurs et les concepteurs trouvent souvent des difficultés à les mettre en ouvre particulièrement pour des configurations, des chargements, et des conditions aux limites relativement complexes; cependant les méthodes numériques sont incontestablement l'outil d'analyse structurelle le plus puissant et le plus fiable dans ce sens. Parmi ces outils numériques on peut citer, la plus populaire la méthode des éléments finis (MEF), la méthode des bandes finies (MBF), la méthode des bandes finies contraintes $(\mathrm{MBFc})$, la théorie des poutres généralisées (GBT) ... etc.

En ingénierie de structure la MEF est la plus robuste, car elle offre une manipulation générale des géométries, du chargement et des conditions aux limites, en revanche elle ne fournit pas l'identification modale (la charge critique d'instabilité et la forme déformée correspondante).

La méthode des bandes finies qui est une variante économique de la MEF (DDL réduits), utilise des harmoniques comme fonctions d'interpolation pour approcher le déplacement dans une direction privilégiée. Popularisée par le professeur Hancock, qui l'a mis en œuvre par courbe de signature qui donne la relation entre le facteur de charge $f_{c r} / f_{y}$ et la longueur de demi-onde autrement dit, en forçant la structure à se déformer en développant une demi-sinusoïde telle que le montre la figure 4. La méthode des bandes finies est devenue un outil incontournable dans l'analyse de stabilité des profilés à parois minces soumis à des contraintes longitudinales.
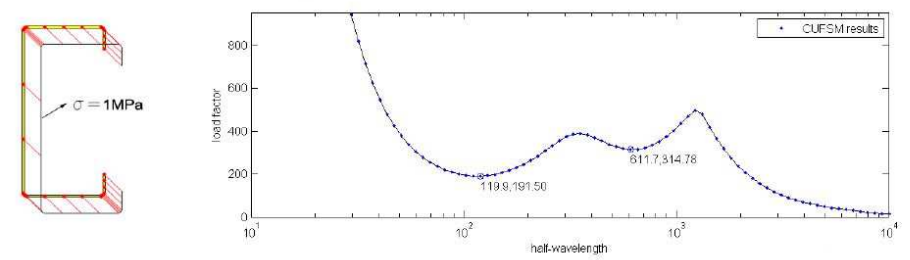

Fig. 4. La courbe signature issue d'analyse par la MBF d'une colonne en $\mathrm{C}$.

\section{L'analyse plastique}

Finalement, il paraît opportun d'indiquer qu'une méthode de conception adoptant une analyse plastique n'est pas incluse dans les codes de calculs, étant donné que la majorité des sections en acier formées à froid présentent des rapports largeur épaisseur qui dépassent considérablement les limites pour une analyse plastique.

\section{Analyse locale et distorsionnelle des profilés en $\mathrm{C}$ à parois minces en acier formé à froid}

Une étude a été mise en œuvre sur un groupe d'échantillons de profilés de section en c comprimées très utilisés comme pannes et lisses de bardage. Cette investigation consiste à estimer la charge critique élastique locale et distorsionnelle par les formules précédentes et par la suite coordonnées ces données aux résultats numériques de la méthode des bandes finies FSM (finite strip method).

Les propriétés géométriques sont résumées dans le tableau 1 sachant que 'h"': est la hauteur de l'âme, 'b" étant la largeur de la semelle, 'c'" : est la profondeur du raidisseur, et ' $t$ '" : est l'épaisseur de la paroi.

Tableau 1. La géométrie des profilés analysés

\begin{tabular}{|l|c|c|c|c|}
\hline & $\mathrm{h} / \mathrm{t}$ & $\mathrm{b} / \mathrm{t}$ & $\mathrm{c} / \mathrm{t}$ & Nbr de sections \\
\hline Min & 44 & 23.33 & 6.67 & \multirow{2}{*}{20} \\
\hline Max & 150 & 46.67 & 13.33 & \\
\hline
\end{tabular}


En outre, les caractéristiques mécaniques de l'acier utilisé sont comme suit $\mathrm{f}_{\mathrm{y}}=140 \mathrm{MPa}, \mathrm{E}=210 \mathrm{GPa}, \mathrm{v}=0.3$.
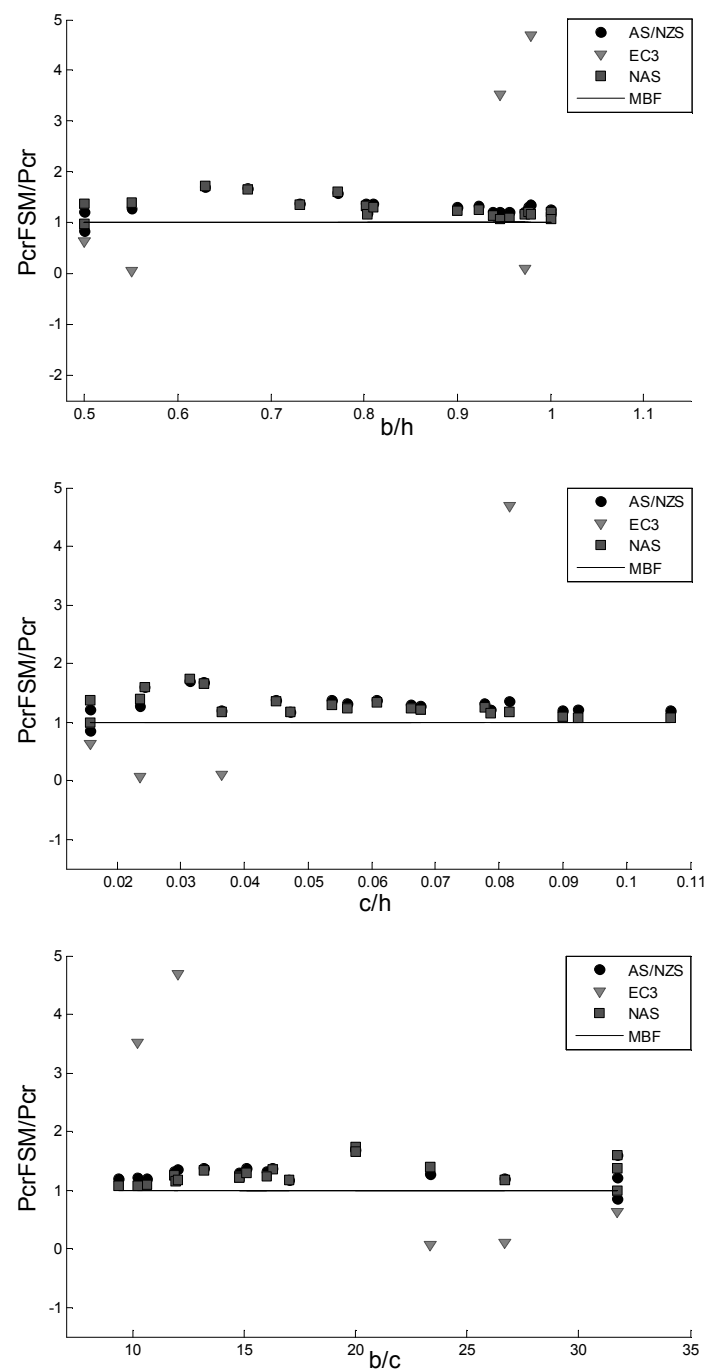

Fig. 5. Comparaison entre les charges critiques distorsionnelles de NAS et ASNZS, EC3 et celles de la MBF pour les rapports $\mathrm{h} / \mathrm{b}, \mathrm{c} / \mathrm{h}, \mathrm{b} / \mathrm{c}$

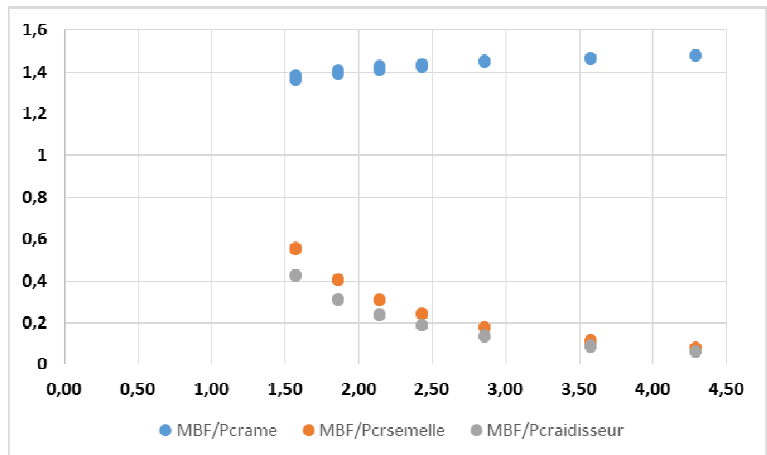

Fig. 6. La performance des trois parois vis-à-vis du mode local évaluée pour le rapport $\mathrm{h} / \mathrm{b}$

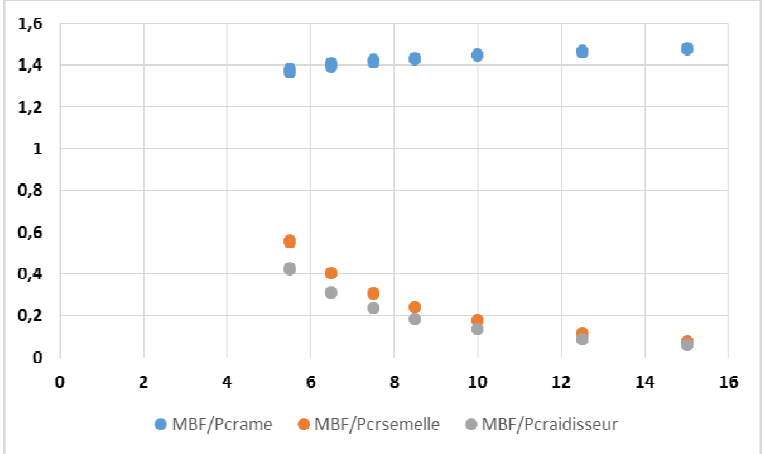

Fig. 7. La performance des trois parois vis-à-vis du mode local évaluée pour le rapport $\mathrm{h} / \mathrm{c}$

\section{Analyse des résultats}

La figure 5 expose le comportement mécanique de la section en fonction de sa géométrie (PcrFSM/PcrAS/NZS, PcrFSM/PcrNAS, PcrFSM/PcrEC3), les modèles américain et australien sont toutes les deux performent vu que le rapport PcrFSM/Pcr NAS ou PcrFSM/Pcr ASNZS sont aux alentours de 1 , autrement dit leur dispersion n'est pas importante par rapport à la méthode numérique. Cependant, le modèle européen, exhibe des résultats peu favorables, pour la majorité des valeurs de $\mathrm{b} / \mathrm{h}, \mathrm{c} / \mathrm{h}$ et $\mathrm{b} / \mathrm{c}$. L'absence de résultats de certaines sections et la divergence des autres se traduisent par une performance de l'Eurocode qui sont jugées très faibles, et cela est justifié par le manque d'adaptation de ce modèle par rapport à ces géométries, et le comportement de ces sections ne représente pas effectivement la schématisation de l'Eurocode du mode distortionnel.

Quant aux figures 6 et 7 les sections qui sont enclines au voilement de la semelle et du raidisseur sont ceux qui possèdent des rapports $\mathrm{h} / \mathrm{b}$ et $\mathrm{h} / \mathrm{c}$ intermédiaires d'où une dispersion minime des charges critiques du voilement de la semelle et le raidisseur par rapport à la MBF, pour des rapports plus importants le voilement de l'âme prend le relais et régit le comportement de la section.

\section{Conclusion}

La résistance des profilés à parois minces est dans la plupart des cas affectée par le mode local et le mode distosionnel. Le présent article a mis en évidence un état de l'art sur les méthodes analytiques et numériques servant à analyser les modes d'instabilité des profilés à parois minces en acier formé à froid, entre autres la performance de ces méthodes a été estimée pour le mode local et distorsionnel, et les conclusions suivantes ont été révélées :

(i) Plus le raidisseur accroît mieux est la résistance au mode distorsionnel

(ii) Les méthodes américaine et australienne donnent des résultats similaires voire identiques et leurs modèles sont fiables et éprouvent une convergence par rapport à la méthode des bandes finies. 
(iii) Plus les rapports $\mathrm{h} / \mathrm{b}$ et $\mathrm{h} / \mathrm{c}$ augmentent moins la contrainte critique du mode local de la semelle et le raidisseur est appréciable.

(iv) Le modèle Européen pour le mode distorsionnel, présente des disfonctionnements pour la majorité des sections.

(v) On pourrait avancer que l'EC3 considère le mode distorsionnel comme une continuité du mode local, et en conséquence les résultats divergent par rapport à ceux du deuxième minimum de la courbe signature de la MBF.

\section{Références}

1. 4600:2005, AS/NZS. Australian/New Zealand Standard Cold-Fomred Steel Structures. s.1. : standards australia standards new zealand, (2005).

2. Institute, American and Iron Steel. AISI S100-2007, North American Specification for the Design of ColdFormed Steel Structural Members .(2007).

3. Distortional Buckling of Steel Storage Rack Columns. Hancock, Gregory J. St. Louis, Missouri, U.S.A. : s.n., November 13-14, (1984). Seventh International Specialty Conference on Cold-Formed Steel Structures.

4. Cold-formed steel structures: Research review (2013-2014). Hancock, GJ. 2016, Advances in Structural Engineering, Vol. 19(3), pp. 393-408.

5. EN1993-1-3, European Standard pr. EC3. Eurocode 3: design of steel structures. . s.1. : European Committee for Standardization, (2007).

6. Effective Width Method for determining distortional buckling strength of cold-formed steel flexural $C$ and $Z$ sections. Cheng Yu, Weiming Yan. (2011), THINWALLED STRUCTURES, Vol. 49, pp. 233-238.

7. AISI-Specifications for the Design of Cold-Formed, Steel Structural Members. AISI Manual Cold-Formed Steel Design. s.l.: American Iron and Steel Institute, (2002). 